\title{
Lp-Pla2 Selective Inhibitor (Darapladib) Effect in Lowering Insulin Resistance and Aortic tissue Inflammation at type 2 Diabetes Mellitus
}

\author{
Titin Andri Wihastuti ${ }^{1}$, Dinda Zahra Putri Andiyani ${ }^{1}$, Sri Andarini ${ }^{1}$, Teuku Heriansyah ${ }^{2 *}$ \\ ${ }^{1}$ Department of Biomedicine, Faculty of Medicine, Brawijaya University, Malang, Indonesia. \\ ${ }^{2}$ Department of Cardiology and Vascular Medicine, Faculty of Medicine, Syiah Kuala University, Banda Aceh, Indonesia.
}

\begin{tabular}{|c|c|}
\hline ARTICLE INFO & ABSTRACT \\
\hline Article history: & \multirow{9}{*}{ 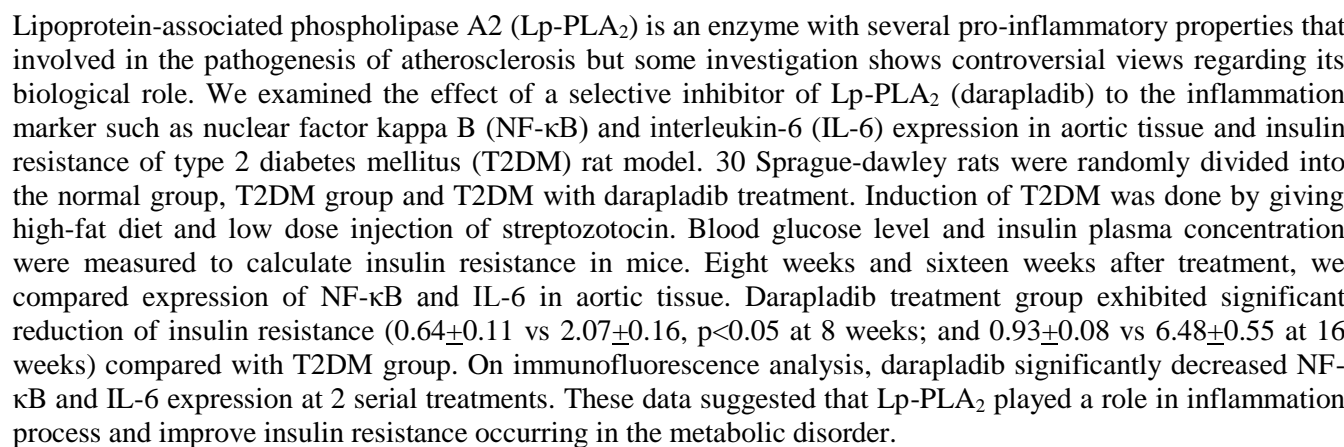 } \\
\hline Received on: $06 / 03 / 2017$ & \\
\hline Accepted on: 06/11/2017 & \\
\hline Available online: $30 / 12 / 2017$ & \\
\hline Key words: & \\
\hline Type 2 Diabetes Mellitus, & \\
\hline Inflammation, Insulin & \\
\hline & \\
\hline Darapladib. & \\
\hline
\end{tabular}

\section{INTRODUCTION}

Type 2 diabetes mellitus (T2DM) is one of the most non-communicable diseases involving multiple genes and environmental factors that characterized by impaired insulin secretion through a dysfunction of pancreatic $\beta$-cells and insulin resistance (Ozougwu et al., 2013). It is associated with an increased risk of atherosclerotic cardiovascular disease (Niskanen et al., 1998). Prolonged exposure to hyperglycemia altered vascular homeostasis and associated with vascular inflammation, that has been hypothesized underlying atherosclerosis process in T2DM condition (Kim et al., 2010).

\footnotetext{
* Corresponding Author

Teuku Heriansyah, Department of Cardiology and Vascular Medicine, Faculty of Medicine, Syiah Kuala University, Banda Aceh Faculty of Medicine, Syah Kuala University, St. Teuku Nyak Arief Darussalam, Banda Aceh, Aceh, Indonesia.

Phone: 6281-1661-8282,Email: teuku_hery@ unsyiah.ac.id
}

Both free fatty acid and AGE directly activate nuclear factor- $\mathrm{kB}(\mathrm{NF}-\mathrm{\kappa B})$ that required for the transcription of most proinflammatory molecules, adhesion molecules, cytokine in the pathology of atherosclerosis and diabetic complication (Patel and Santani, 2009). Study showed that interleukin-6 (IL-6) level are used to predict risk of cardiovascular disease in the development of T2DM because it had a role in the pathogenesis of insulin resistance (Hameed et al., 2015).

The secreted plasma form of platelet-activating factor acetylhydrolase (PAF-AH) also known as lipoprotein-associated phospholipase $A_{2}$ (Lp-PLA $A_{2}$ is an enzyme circulates in the blood and found in atherosclerotic lesion. This enzyme has a dualism since it has anti- inflammatory properties to hydrolyze proinflammatory glycerophospholipid PAF, and has pro-inflammatory properties since its substrate generates lysophosphatidylcholine and oxidized fatty acid. Lp-PLA 2 's pathways that related to inflammation has been hypothesized to have a rolein atherosclerosis process. 
Darapladib is a reversible Lp-PLA 2 inhibitor that has been extensively tested in vitro and in vivo (Stafforini and Zimmerman, 2014). However, studies show controversial results about Lp-PLA,'s role in cardiovascular disease (Hassan, 2015). So, this study aims to determine the role of $\mathrm{Lp}-\mathrm{PLA}_{2}$ selective inhibitor to the expression of inflammation marker such as NF- $\kappa \mathrm{B}$ and IL-6 in the T2DM rat model.

\section{MATERIAL AND METHODS}

The study was carried out in the Central Laboratory of Biological Sciences, Brawijaya University Malang, Indonesia. The investigation was conducted according to the principle expressed in the 'Guiding Principles on the Care and Use of Animals'. The study received prior approval from the ethics committee of the Animal Care and Use Committee Brawijaya University Number 400/EC/KEPK/10/2016 at October, $13^{\text {th }} 2016$.

\section{Animals}

\section{Acclimatization phase}

Thirty male, six-week-old, Sprague-Dawley rats (Bogor Agricultural University, Indonesia), 150-200 gram weight each experienced acclimatization phase during two weeks adjustment period. All animals were given normal diet consist of comfeed PARS, wheat flour and water as much as 26 gram/rats/day. The rest amount of feed was taken and weighed every 24 hours. After the acclimatization phase, then rats continued to the randomization process to be divide into treatment phase.

\section{Treatment phase}

In this phase, Sprague-dawley rats were divided into the negative control (normal) group, positive control group (T2DM) and study group (T2DM with darapladib treatment). Each group was divided into 2 serial treatments: 8 weeks and 16 weeks. The negative control group was given normal rats food contained the total energy of $3.43 \mathrm{kcal} / \mathrm{g}$ while T2DM induction was done by giving high-fat diet contained the total energy of $5.29 \mathrm{kcal} / \mathrm{g}$ and also intraperitoneal injection of low dose streptozotocin $30 \mathrm{mg} / \mathrm{kg}$ bw. Darapladib (Glaxo Smith Kline) was given orally $20 \mathrm{mg} / \mathrm{kg}$ bw once daily according to the serial time group given.

\section{Laboratory Method}

Lipid profiles measured total cholesterol, high-density lipoprotein (HDL) cholesterol and low-density lipoprotein (LDL) cholesterol in rat blood serum (EnzyChrom ${ }^{\mathrm{TM}}$ kit). T2DM was diagnosed by measuring fasting blood glucose level one week after streptozotocin injection taken from rat's tail with the result of $>7.0 \mathrm{mmol} / \mathrm{L}$ (GlucoDR blood glucose test-meter All Medicus Co.Ltd, Dongan-gu, Anyang-si, Korea). The insulin level in rat's blood plasma (Rat INS ELISA kit Cat. No. E-EL-R 2466). Insulin resistance was calculated by using homeostatic model assessmentinsulin resistance (HOMA-IR) formula in rat model. HOMA IR's result of $>1.716$ can be categorized as insulin resistance with a sensitivity of $83.87 \%$ and specificity of $80.56 \%$ ):
HOMA-IR = fasting blood glucose $(\mathrm{mmol} / \mathrm{L}) \times$ fasting insulin plasma (IU/L)/14.1 (VanDjik, 2013)

\section{Immunofluorescence Analysis}

Expression of NF- $\mathrm{BB}$ and IL-6 were measured by immunofluorescence staining. Aortic tissue that previously fixed with PHEMO buffer and processed by immunofluorescence labeling with anti-rat antibody IL-6 and fluorescein isothiocyanate (FITC) secondary antibody; and anti-rat antibody NF- $\kappa B p 65$ and HIF-1 $\alpha$ to ensure NF- $\kappa$ B activation in the nucleus(BIOS Inc., Boston, MA, USA).

Those parameters were observed with confocal laser scanning microscope (Olympus Corporation, Tokyo, Japan) and quantitatively analyzed using Olympus FluoView Software (version 1.7A; Olympus Corporation).

\section{Statistical Analysis}

Results are expressed as the mean \pm SD. One-way analysis of variance (ANOVA) test used to determine the difference between the control and the study groups both in 8 weeks and 16 weeks serial time. It was continued by post hoc test using duncan method to detect the differences in each intervention group. The level of statistical significance was defined by $\mathrm{p}<0.05$ (SPSS software version 20 IBM Corporation, New York, NY, USA).

\section{RESULT}

\section{Insulin resistance analysis in darapladib treatment}

Fasting blood glucose, insulin plasma level, and insulin resistance calculation using HOMA-IR formula are shown in figure 1. T2DM group at 8 weeks and 16 weeks serial times have a higher level of fasting blood glucose $(8.46 \pm 0.91$ and $7.89 \pm 0.50)$, higher insulin plasma $(3.48 \pm 0.50$ and $11.60 \pm 1.19)$ and the abnormal result of HOMA-IR $(2.07 \pm 0.16$ and $6.48 \pm 0.55)$ than normal group. Darapladib treatment group have a normal level of fasting blood glucose $(5.46 \pm 0.63$ and $5.44 \pm 0.60)$, insulin plasma level $(1.64 \pm 0.11$ and $2.43 \pm 0.24)$ and HOMA-IR $(0.64 \pm 0.11$ and $0.93 \pm 0.08)$. Mann-Whitney test shows that darapladib treatment can significantly $(\mathrm{p}<0.05)$ normalize fasting blood glucose, insulin plasma level and insulin resistance in the T2DM group at both 8 weeks and 16 weeks serial time.

\section{Lipid profile analysis in darapladib treatment}

Lipid profile result are shown in figure 2. T2DM group at 8 weeks and 16 weeks serial times tend to have a higher level of total cholesterol $(123.00 \pm 2.86$ and $111.72 \pm 7.30)$, higher level of LDL-cholesterol(95.53 \pm 8.66 and 88.24 \pm 6.23$)$, and a lower level of HDL-cholesterol $(4.96 \pm 0.41$ and $13.96 \pm 0.87)$ than normal group. Mann -Whitney test show that darapladib treatment at 8 weeks and 16 weeks serial time can significantly $(\mathrm{p}<0.05)$ normalize total cholesterol $(97.96 \pm 1.70$ and 98.96 \pm 3.24$), \quad$ LDL-cholesterol $(85.92 \pm 6.84$ and $61.52 \pm 6.03)$ and HDL-cholesterol $(16.44 \pm 1.24$ and $20.80 \pm 2.76)$ near to the normal group. 


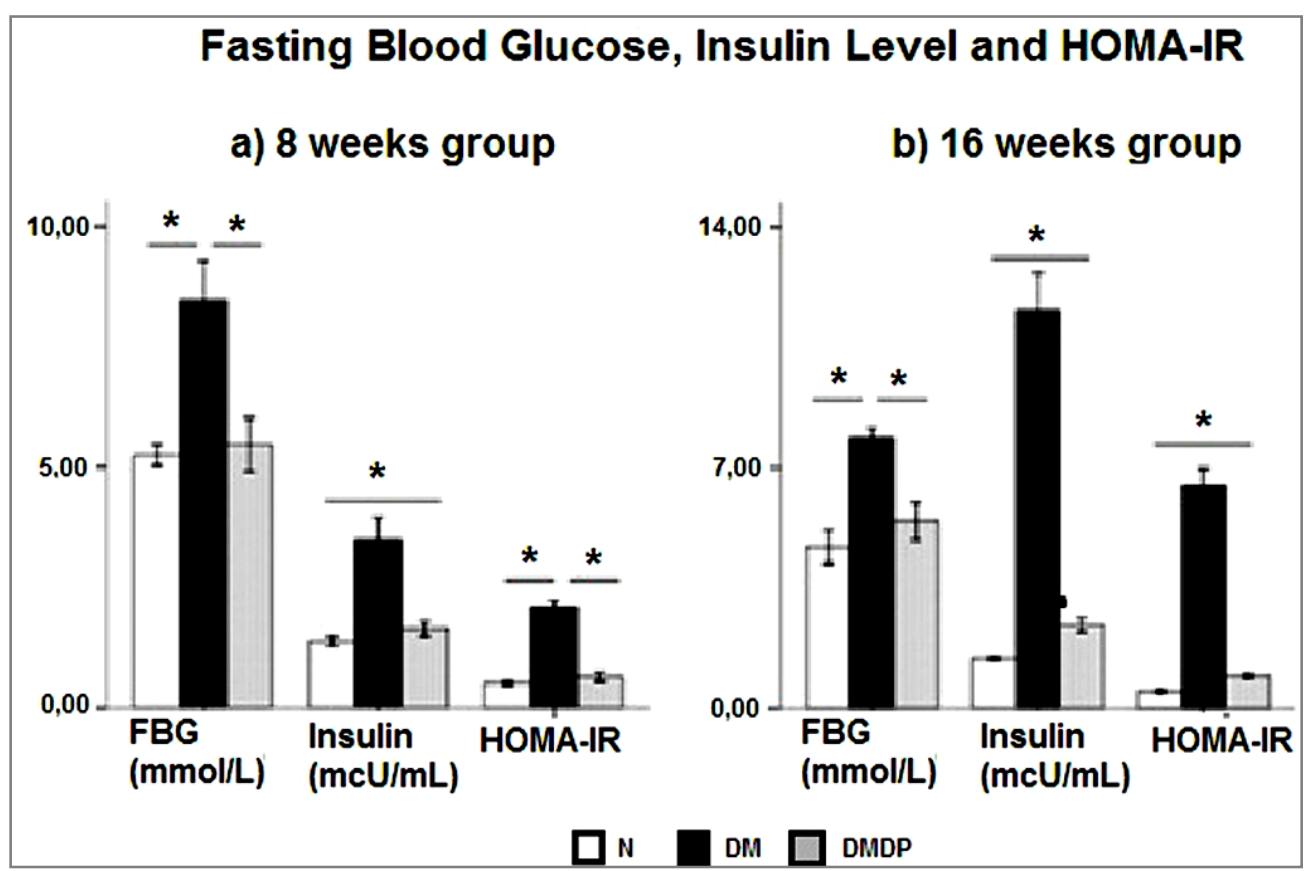

Fig. 1: Effect of darapladib treatment on fasting blood glucose, insulin plasma and insulin resistance level at 8 weeks and 16 weeks treatment. Mann whitney test showing significant result $(*)$ at $\mathrm{p}<0.05$. N: normal group, DM: Type 2 diabetes mellitus group, DMDP: type 2 diabetes mellitus with darapladib treatment.

\section{Lipid Profile}

a) 8 weeks group

b) 16 weeks group
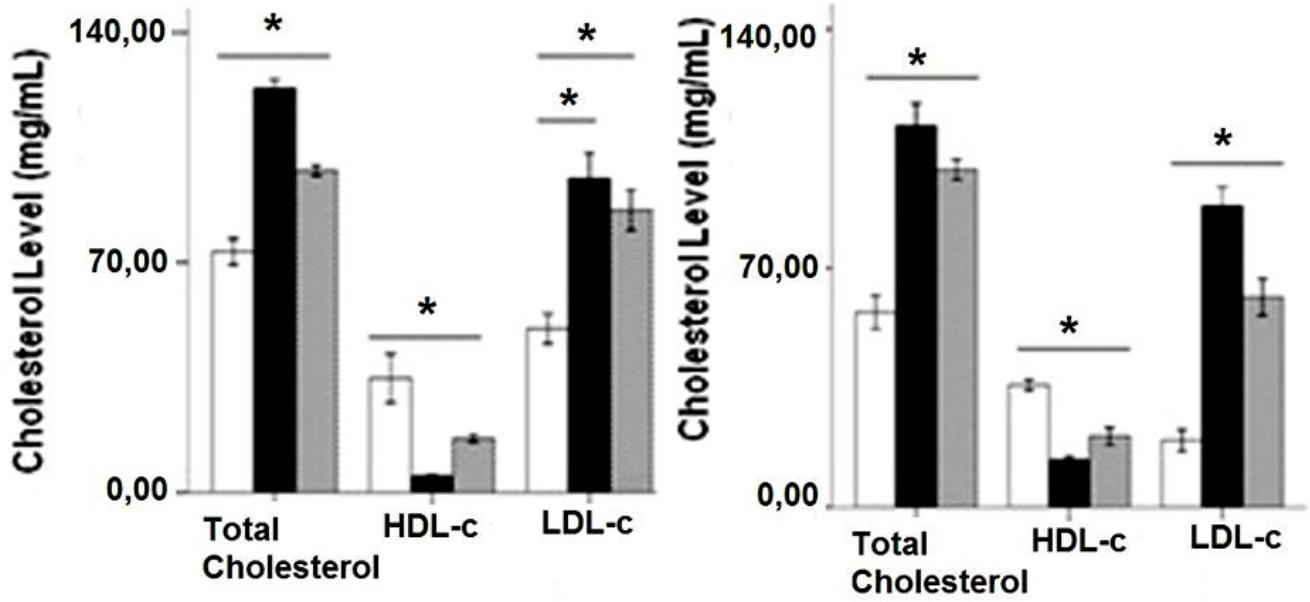

N DDM DDMP

Fig. 2: Effect of darapladib treatment on total cholesterol, HDL cholesterol and LDL cholesterol level at 8 weeks and 16 weeks treatment. Mann whitney test showing significant result $(*)$ at $\mathrm{p}<0.05 . \mathrm{N}$ : normal group, DM: Type 2 diabetes mellitus group, DMDP: type 2 diabetes mellitus with darapladib treatment.

Immunofluorescence analysis

Mean inflammation rate are shown in figure 3. T2DM group tends to have a higher level of NF- $\kappa \mathrm{B}$ and IL-6 expression whereas darapladib treatment group have a lower level near to the normal one. One way ANOVA test with post hoc Tukey method shows a significant value $(\mathrm{p}<0.05)$ for darapladib in lowering NF$\kappa \mathrm{B} \&$ IL- 6 expression of aortic tissue at 2 serial time treatments (figure $3 \mathrm{a}$ and $3 \mathrm{c}$ ).Immunofluorescence staining using rhodamine secondary antibody for IL-6 expression and double staining with FITC and rhodamine secondary antibody for NF- $\mathrm{KB}$ activation show qualitative expressions of specific inflammation marker at the aortic tissue. Figure (3b) and (3d) show that T2DM group has maximum fluorescence intensity while normal and darapladib group has low-medium fluorescence intensity. 
a) NF-kB activation

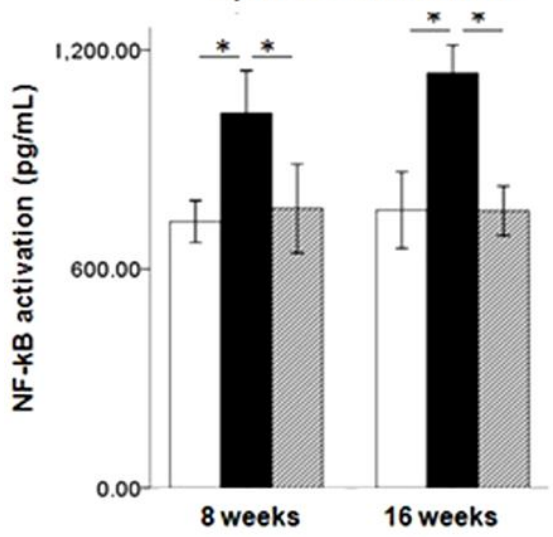

c) IL-6 expression

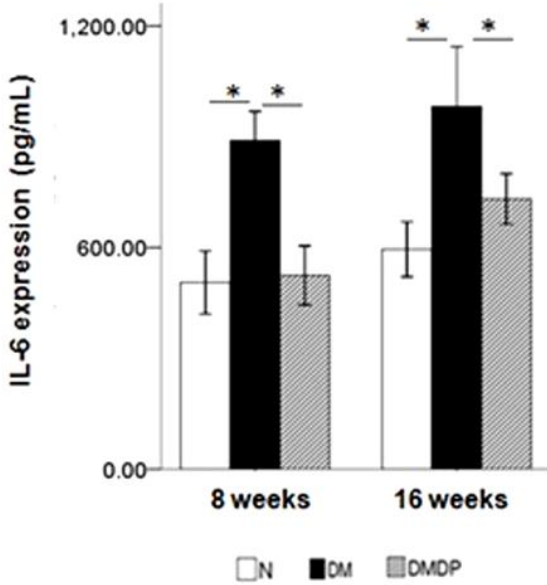

b) NF-kB activation on immunofluorescence

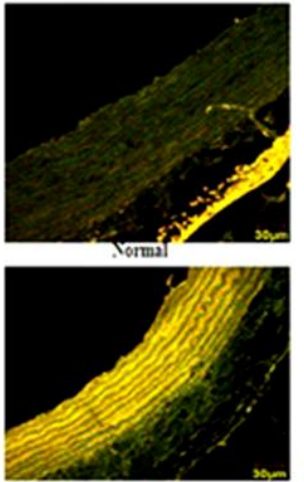

T2DM

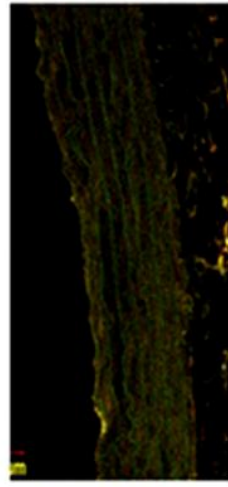

I:DM-Darapladib d) IL-6 expression on immunofluorescence

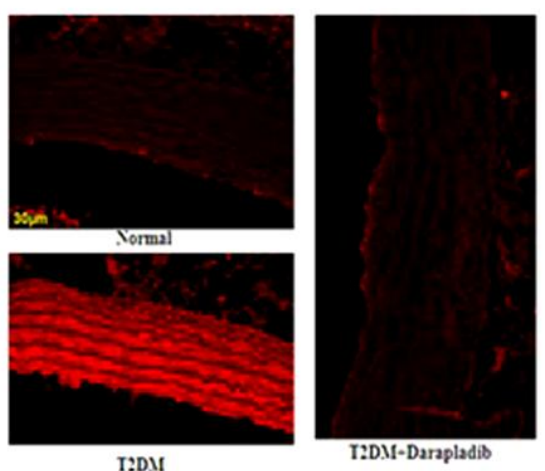

Fig. 3: Immunofluorescence results showing cytokine expression in aortic tissue (400x). N: normal group, DM: Type 2 diabetes mellitus group, DMDP: type 2 diabetes mellitus with darapladib treatment. (A) Mean value of quantification result in NF- $\kappa \mathrm{B}$ expression. NF- $\kappa \mathrm{B}$ was significantly inhibited in darapladib group both at 8 weeks and 16 weeks serial treatment ( $\mathrm{p}=0.044$ and $\mathrm{p}=0.001$ vs T2DM group); (B)Double staining rhodamine and FITC secondary antibody (yellow color) show NF-KB expression in the nuclues. Normal and darapladib group has dim intensity than T2DM group; (C) Mean value of quantification results in IL-6 expression. IL-6 was significantly inhibited in darapladib group both at 8 weeks and 16 weeks serial treatment ( $\mathrm{p}=0.025$ and $\mathrm{p}=0.001 \mathrm{vs}$ T2DM group); (D) Rhodamine secondary antibody (red color) show IL-6 expression. Normal and darapladib group has dim intensity than T2DM group

\section{DISCUSSION}

\section{Lipid Profile and Insulin Resistance in T2DM group and Darapladib Treatment Group}

Type 2 diabetes mellitus (T2DM)was associated with increased risk of cardiovascular disease with hypothesis said insulin resistance, hyperglycemia, dyslipidemia and inflammatory processes have an important role in every step of atherosclerotic plaque formation and rupture (Ziegler, 2005). Inflammation plays a key role in T2DM pathogenesis and is related to the increase in cardiovascular disease risk. Hyperglycemia and dyslipidemia that occur in T2DM stimulate the endothelial inflammation response (Mazzone et al., 2008).

Lipid metabolism becomes dysregulated in a variety of diseases, including obesity and diabetes. Dyslipidemia that occurred in mice that were given HFD and STZ was similar to the metabolic profiles in individuals with type 2 diabetes (Skovso, 2014). Weight gain and the occurrence of dyslipidemia are predisposing factors of obesity that plays a major role in insulin resistance (Shaw et al., 2010). The buildup of toxic metabolites of lipids (fatty acyl CoA, diacylglycerol and ceramide) in skeletal muscle, liver adipocytes, beta cells, and arteries contribute to the development of insulin resistance, beta cell dysfunction and atherosclerosis (Reaven, 2005). Animal models of type 2 diabetes mellitus given a combination of a high-fat diet experienced hyperinsulinemia, insulin resistance and/or glucose intolerance, followed by STZ as beta cell toxin that caused degeneration of functional beta cells. Both of these stressors are designed to resemble the pathology of type 2 diabetes in human in a shorter amount of time (Zhang et al., 2008).

Administration of darapladib in T2DM group had significantly different parameters compared to the diabetic group. There was an improvement in lipid profile values and insulin resistance with darapladib treatment. There are several mechanisms that link Lp-PLA2 with diabetes, inflammation state associated with an increase ofLp-PLA2 activity in hydrolyzing 
oxidized phospholipids induce insulin resistance and increase type 2 diabetes mellitus risk.

The increase in Lp-PLA2 is considered as a marker of inflammation in adipose tissue and the increase of fatty acids or adipokine flow that promote insulin resistance and pancreatic beta cell failure (Nelson et al., 2012). Many studies associated LpPLA2 with cardiovascular disease, but only a few studies established the relationship of Lp-PLA2 activity with diabetes, especially type 2 diabetes mellitus.

\section{Inflammation Marker in T2DM group and Darapladib Treatment Group}

NF-kB transduction signaling pathway in aortic endothelium is a process that happens in the early stage of atherosclerosis lesion formation (Hajra et al., 2000). NF-kB activation leads to the activation of several genes that play a role in the pathogenesis of the formation of atherosclerosis lesion (Patel and Santani, 2009). NF- $\mathrm{B}$ plays a crucial role in vascular physiopathology so it participates various complication associated with DM through its role to induce systemic inflammation.NF- $\kappa \mathrm{B}$ reflects inflammatory state at the aortic tissue and NF- $\kappa \mathrm{B}$ is correlated with impaired of blood glucose level, evidence of dyslipidemia, the progression of an inflammatory state, vasodilatation and insulin resistance (Indira and Ahilash, 2013).

Endothelial dysfunction is the first phenomenon happened in line with insulin resistance that contributes to the pathogenesis of cardiovascular disease, which interleukin-6 (IL-6) is proinflammatory cytokine correlates with those abnormalities (Esteve et al., 2007). Studies show that circulating IL-6 was directly related to endothelial dysfunction and insulin resistance, that can be some kind of predictor to the development of T2DM and cardiovascular events (Pradhan et al., 2001; Shinohara et al., 2012). Prolonged tissue injury because of hyperglycemia state increase inflammatory cytokine, such as TNF- $\alpha$ and IL-6 that activate inflammatory cascade mediated by cellular adhesion molecules in endothelial cells called endothelial activation (Antonaides et al., 2004). Anti-inflammatory substances are given to diabetics may restore glucose level and diminish risk of cardiovascular events (Tabit et al., 2010).

Inhibitor of IkB kinase (IKK) signaling pathway is one of many key mechanisms that is activated by oxidative stress and inflammation, and it regulates the cytokines that control the process of cellular apoptosis, cellular cycle progression, and inflammation (Tilstra et al., 2011). NF- $\kappa \mathrm{B}$ is activated by several factors that become abnormal in type 2 diabetes mellitus patients, such as glucose, fatty acids, advanced glycation end product, and toll-like receptor. NF- $\kappa \mathrm{B}$ is the final pathway that connects all of the activated inputs in type 2 diabetes mellitus that eventually leads to atherosclerosis response. $\mathrm{NF}-\kappa \mathrm{B}$ regulates several molecules that plays some roles in diabetic atherosclerosis processes, such as TNF- $\alpha$, IL- 1 and IL- 6 which can worsen the insulin resistance, TLR and resistin.

$\mathrm{NF}-\kappa \mathrm{B}$ regulator proteins such as inhibitor $\kappa \mathrm{B}(\mathrm{I} \kappa \mathrm{B})$ and distal targets such as c-jun $\mathrm{N}$-terminal kinase (JNK) are associated with insulin sensitivity and atherosclerosis (Indira et al., 2013). NF-kB activation is the process of NF-kB subunit translocation from cytoplasm to nucleus and stimulates the expression of proinflammatory genes such as cytokines (TNF- $\alpha$ and interleukin), adhesion molecules (ICAM-1 and VCAM-1) and chemokines (Wihastuti et al., 2016). Activation of NF-kB initiates atherosclerosis process and endothelial dysfunction is characterized by an increase in iNOS, platelet adhesion, and migration and proliferation of smooth muscle cells (Wihastuti et al., 2014). Signs of inflammation may occur at any time, from the initial to the later stage of atherosclerosis development (Heriansyah et al., 2016). AGE may activate vascular smooth muscle cell NF- $\kappa \mathrm{B}$ and mediate the migration and proliferation of smooth muscle cells (Tabit et al., 2010).

In this study, there was an increase of NF-kB and IL-6 expression in type 2 diabetes mellitusrat aorta. Increased expression of NF- $\kappa \mathrm{B}$ because of cytokines, high glucose levels, and AGE explains the occurrence of vascular dysfunction in diabetes, and the activation of $\mathrm{NF}-\kappa \mathrm{B}$ can enhance the activation of important genes involved in the pathology of atherosclerosis (Patel and Santani, 2009).

Lp-PLA2 appeared as an effective marker in detecting cardiovascular disease. Macrophages that infiltrate adipose tissue increase the production of Lp-PLA2.Lp-PLA2 induces the expression of several pro-inflammatory cytokines through the accumulation of its product in the form of lysoPC. Lp-PLA2 activity is also associated with oxidative stress events through its activities in modifying phospholipids. Free radicals under hyperglycemia conditions play a role in increasing lipoprotein oxidation process; thereby increasing Lp-PLA2 substrates. In another word, Lp-PLA2 helps to increase the severity of inflammation, oxidative stress, and microvascular-dysfunction mediating the pathogenesis of cardiovascular disease, especially atherosclerosis in type 2 diabetes mellitus (Zhu, 2016).

NF-kB and MAPK pathways are closely associated with inflammatory responses in cells. NF-kB and MAPK pathways were related to the regulation of Lp-PLA2. NF-kB signaling pathway could interfere with insulin signaling through an inhibition of insulin receptor substrate 1, Akt (protein kinase B), and eNOS phosphorylation, and lead to a decrease in NO production, greater incidence of inflammation and endothelial dysfunction (Han et al., 2015).

Therapeutic strategies using antibodies or molecules that target IKK-b-NFkB (salicylates, salsalate), TNF- $\alpha$ (etanercept, infliximab, adalimumab), IL-1 $\beta$ (anakinra) and IL-6 (tocilizumab) to cure the inflammation may improve insulin sensitivity and beta cell function (Esser et al., 2015). This study proved that the effect of darapladib as a selective inhibitor of Lp-PLA2 in reducing aortic NF-kB and IL6 expression may affect insulin sensitivity; this was proved by the occurrences of normoglycaemia, normoinsulinemia, and normal HOMA-IR level and lipid profile in the treatment group of type 2 diabetes Sprague Dawley rats models. 


\section{CONCLUSION}

This study proves the role of darapladib as antiinflammatory properties to diminish NF- $\kappa$ B and IL-6 levels and improve insulin resistance in the T2DM rat model.

Financial support and sponsorship: Ministry of Research, Technology and High Education.

Conflict of Interests: There are no conflicts of interest.

\section{REFERENCE}

Antoniades C, Tousoulis D, Tountas C, Tentolouris C, Toutouza $\mathrm{M}$, Vasiliadou $\mathrm{C}$ et al. Vascular endothelium and inflammatory process, in patients with combined type 2 diabetes mellitus and coronary atherosclerosis: the effects of vitamin C. Diabet Med. 2004; 21(6): 552-8. Doi: 10.1111/j.1464-5491.2004.01201.x

Esser N, Paguot N, Scheen AJ. Anti-inflammatory agents to treat or prevent type 2 diabetes, metabolic syndrome and cardiovascular disease. Expert Opin Investig Drugs. 2015; 24(3): 283-307

Esteve E, Castro A, Bermejo AL, Vendrell J, Ricart W, Real JM. Serum interleukin-6 correlated with endothelial dysfunction in healthy men independently of insulin sensitivity. Diabetes Care. 2007 Apr; 30(4): 939-45. 10.2337/dc06-1793.

Hajra L, Evans AI, Chen M, Hyduk SJ, Collins Tm Cybulsky MI. The NF- $\mathrm{KB}$ signal transduction pathway in aortic endothelial cells is primed for activation in regions predisposed to athersclerotic lesion formation. PNAS. 2000; 97(16): 9052-7.

Hameed I, Masoodi SR, Mir SA, Nabi M, Ghazanfar K, Ganai BA. Type 2 diabetes mellitus: from a metabolic disorder to an inflammatory condition. World J Diabetes. 2015; 6(4): 598-612. Doi: 10.4239/wjd.v6.i4.598

Han X, Wang T, Zhang J, Liu X, Li Z, Wang G. Apolipoprotein $\mathrm{CIII}$ regulates lipoprotein-associated phospholipase A2 expression via the MAPK and NFkB pathways. Biology Open. 2015; 4: 661-5.

Hassan M. 2015. Stability and SOLID-TIMI 52: Lipoprotein associated phopholipase $\mathrm{A}_{2}\left(\mathrm{Lp}-\mathrm{PLA}_{2}\right)$ as a biomarker or risk factor for cardiovascular disease.

Heriansyah T, Adam AA, Wihastuti TA, Rohman MS. Elaborate evaluation of serum and tissue oxidized LDL level with darapladib therapy: a feasible diagnostic marker for early atherogenesis. Asian Pacific Journal of Tropical Biomedicine. 2016. Doi: 10.1016/j.apjtb.2016.11.014

Indira M, Abhilash PA. Role of NF-кappa B (NF-kB) in diabetes. Forum Immun Dis Ther. 2013; 4(2): 111-32. DOI: 10.1615/ForumImmunDisTger.2013008396

Kim TN, Kim S, Yang SJ, Yoo HJ, Seo JA, Kim SG et al. Vascular inflammation in patients with impaired glucose tolerance and type 2 diabetes: analysis with ${ }^{18} \mathrm{~F}$-Fluorodeoxyglucose positron emission tomography. $2010 ; \quad 3: \quad 142-8$. http://dx.doi.org/10.1161/CIRCIMAGING.109.888909.

Mazzone T, Chait A, Plutzky J. Cardiovascular disease risk in type 2 diabetes mellitus: insights from mechanistic studies. Lancet. 2008; 371(9629):1800-9. Doi: 10.1016/S0140-6736(08)60768-0

Nelson TL, Biggs ML, Mukamal KJ. Lipoprotein associated phospholipase A2 (Lp-PLA2) and future risk of type 2 diabetes: results from the cardiovascular health study. J Clin Endocrinol Metab. 2012; 97(5): 1695-1701.

Niskanen L, Turpeinen A, Penttila I, Uusitupa MI. Hyperglycemia and compositional lipoproteina bnormalities as predictors of cardiovascular mortality in type 2 diabetes: a 15 -year follow-up from the time of diagnosis. 1998; 21(11): 1861-9. http://dx.doi.org/10.2337/diacare.21.11.1861.
Ozougwu JC, Obimba KC, Belonwu CD, Unakalamba CB. The pathogenesis and pathophysiology of type 1 and type 2 diabetes mellitus. 2013; 4(4): 46-57. Doi:10.5897/JPAP2013.0001.

Patel S, Santani D. Role of NF- $\mathrm{KB}$ in the pathogenesis of diabetes and its associated complication. Pharmacological reports. 2009; 61: 595-603.

Pradhan AD, Manson JE, Rifai N. C-reactive protein, interleukin 6 and risk of developing type 2 diabetes mellitus. JAMA. 2001; 286(3): 327-34. Doi: 10.1001/jama.286.3.327

Reaven G. Insulin Resistance, type 2 diabetes mellitus, and cardiovascular disease. Circulation. 2005; 112: 3030-2.

Shaw JE, Sicree RA, Zimmet PZ. Global estimates of the prevalence of diabetes for 2010 and 2030. Diabetes Res Clin Pract. 2010; 87(1): 4-14. doi: 10.1016/j.diabres.2009.10.007.

Shinohara T, Takahashi N, Okada N, Ayabe R, Kondo H, Yufu $\mathrm{H}$ et al. Interleukin- 6 as an independent predictor of future cardiovascular events in patients with type-2 diabetes without structural heart disease. $\mathbf{J}$ Clin Exp Cardiolog. 2012; 3: 209. Doi: 10.4172/2155-9880.1000209.

Skovso S. Modeling type 2 diabetes in rats using high fat diet and streptozotocin. JDI. 2014; 5(4): 349-58. Doi: 10.1111/jdi.12235

Stafforini DM, Zimmerman GA. Unraveling the PAF-AH/Lp$\mathrm{PLA}_{2}$ controversy. J Lipid Res. 2014; 55(9): 1811-4. Doi:10.1194/jlr.E052886.

Tabit CE, Chung WB, Hamburg NM, Vita JA. Endothelial dysfunction in diabetes mellitus: molecular mechanisms and clinical implications. Rec Endocr Metab Disord. 2010; 11(1): 61-74. Doi: 10.1007/s11154-010-9134-4

Tilstra JS, Clauson CL, Niedernhofer LJ, Robbins PD. NF-kB in aging and disease. Aging Dis. 2011; 2(6): 449-65.

VanDjik TH, Laskewitz AJ, Grefhorst A, Boer TS. A novel approch to monitor glucose metabolism using stable isotopically labelled glucose in longitudinal studies in mice. Lab Anim. 2013; 47(2): 79-88.

Wihastuti TA, Heriansyah T, Soraya M, Wijayanti MD, Firani NK, Iskandar A et al. Inhibition of oxidative stress in hypercholesterolemic rats by soy milk. J Cardiovasc Disease Res. 2016; 7(2):74-82. Doi:10.5530/jcdr.2016.2.4

Wihastuti TA, Sargowo D, Tjokroprawiro A, Permatasari N, Widodo MA, Soeharto S. Vasa vasorum anti-angiogenesis through $\mathrm{H} 2 \mathrm{O} 2$, HIF-1a, NF-kB and iNOS inhibition by mangosteen pericarp ethanolic extract (Garcinia mangostana Linn) in hypercholesterol-diet-given Rattus norvegicus Wistar strain. Vasc Health Risk Manag. 2014; 10: 523-31. Doi:10.2147/VHRM.S61736

Zhang M, Lv XY, Li J, Xu ZG, Chen L. The characterization of high fat diet and multiple low dose streptozotocin induced type 2 diabetes rat model. Experimental Diabetes Research. 2008 ; 2008: 1-9

Zhu HA. Lp-PLA2, a novel potential biomarker predicting cardiovascular disease in type 2 diabetes mellitus. Medical \& clinical review. 2016; 2(2): 20

Ziegler D. Type 2 diabetes as an inflammatory cardiovascular disorder. Curr Mol Med. 2005; 5(3): 309-22.

\section{How to cite this article:}

Wihastuti TA, Andiyani DZP, Andarini S, Heriansyah T. Lp-Pla2 Selective Inhibitor (Darapladib) Effect in Lowering Insulin Resistance and Aortic tissue Inflammation at type 2 Diabetes Mellitus. J App Pharm Sci, 2017; 7 (12): 110-115. 